\title{
Meeting the need: capacity building and social responsibility in neurosurgery
}

\author{
Laura Lippa $^{1}$ (D) Angelos Kolias ${ }^{2}$ \\ Received: 29 February 2020 / Accepted: 4 March 2020 /Published online: 13 March 2020 \\ (C) Springer-Verlag GmbH Austria, part of Springer Nature 2020
}

The article by Uche et al. [17] describes the successful experience of a twinning model for neurosurgical capacity building developed as a collaboration between the Neurosurgery Unit at the University of Nigeria Teaching Hospital and the Swedish Neurosurgery Society. A 2-year trial phase of the prospective study is narrated; the authors take us through a detailed multistep twinning paradigm tailored to address specific needs and overcome specific weaknesses.

Brain tumors, which were the topic of this pilot phase, present unique challenges in low-medium income countries (LMICs) ranging from limited resources to misinformation and lack of literacy, transportation, and lack of health coverage. [7] The paucity of surgeons is distressing: 1 neurosurgeon every 6.2 million people is the mean projection in SubSaharan Africa [5] and the article aligns with this statistic; Uche et al. describe a catchment area of 30 million people with 30 dedicated neurosurgical beds, served by 5 neurosurgeons and 10 residents. The results depicted are an example of what can be achieved: patients in their 30s treated for conditions that would have been otherwise probably left unmanaged, residents exposed to procedures locally uncommon, and formal neurosurgical training provided to 30 nurses, an example of research while most of data in LMICs is unrecognized and unpublished to this day [3], all while dealing with limited health budget and an unstable political situation [4].

Workforce expansion plays a key role in surgical system strengthening; several approaches have been proposed and

This article is part of the Topical Collection on Neurosurgery general

Laura Lippa

laura.lippa@gmail.com

1 Neurosurgery Department, Ospedali Riuniti di Livorno, Livorno, Italy

2 Division of Neurosurgery, Department of Clinical Neurosciences, Addenbrooke's Hospital \& University of Cambridge, Cambridge, UK applied to overcome lack of neurosurgical care worldwide, prioritizing task sharing over task shifting $[4,11,14,16]$; multiple twinning projects between institutions are reported in literature, plus programs and dedicated tenure tracks in the discipline of global surgery. We applaud the systematic and carefully planned efforts of the authors in establishing a locally contextualized partnership between a LMIC Institution and a high-income country (HIC) National Society, allowing access to a broader spectrum of neurosurgical subspecialties than the ones traditionally associated with global neurosurgery (trauma, hydrocephalus, infections).

Since the establishment of WFNS Training Centers, we have seen an increase in number of neurosurgeons especially in SubSaharan Africa, with many of them having already returned to their countries and training surgeons locally $[7,8,16]$.

Donated equipment, which was a key deliverable of the twinning project, allows expansion of the scope and improved quality of neurosurgical practice; however, reliable equipment and ongoing maintenance are issues to tackle [18, 21]. Further missions from the Swedish African Neurosurgical Collaboration (SANC) are already planned, and we are looking forward to read about the long-term impact of this collaboration in the future.

Organizing and attending conferences and workshops in LMICs can help improve professional links and increase local attendance. It also eliminates visa issues so commonly encountered by many LMIC global health practitioners who wish to travel to HICs. [19] In addition to in-person meetings, platforms such as InterSurgeon can help establish international collaborative partnerships tailed on specific needs and offerings of competences. [6]

Partnerships such as the one described in the article can be a vital boost for robust methodological research. Only $4.1 \%$ of global health research activity is surgical and, of all surgical research worldwide, only $4.3 \%$ is relevant to underserved populations. [12] We regard research capacity building in LMICs as an essential component of global surgery efforts, efforts such as the NIHR Global Health Research Group on Neurotrauma are working towards this goal. [9] 
In 2015, an estimated 5 billion people lacked adequate access to essential surgical and anesthesia care, mostly in LMICs and an estimated"143 million additional surgical procedures are needed in LMICs each year to save lives and prevent disability" [10]. Each year, 5 million essential neurosurgical cases are unmet in LMICs and around 23,000 additional neurosurgeons are needed to address them. $[2,13]$

These staggering figures call for a concerted effort by all stakeholders and neurosurgical leaders.

In the past several years, the WFNS and the continental neurosurgical societies have been instrumental in shaping the global neurosurgery efforts. These efforts started taking shape with the 2016 Bogota Statement on Global Neurosurgery and culminated in the creation of the WFNS Global Neurosurgery Committee [20]. This new WFNS committee aims to "align and coordinate all global efforts to collectively address the unmet neurosurgical needs of our patients, whenever and wherever" with the ultimate aim of achieving "universal access to neurosurgical care". The objectives of this committee are the five A's.

1. AMPLIFY access to neurosurgical care.

2. ALIGN all global neurosurgery activity.

3. ADVANCE relevant research, especially from authors in LMICs.

4. ASSIMILATE neurosurgical capacity building in surgical system strengthening.

5. ADVOCATE for (neuro) surgical care within Universal Health Coverage $[1,15]$.

It is socially responsible to be advocates for the needs not only of "our patients" but of patients with neurosurgical conditions worldwide. Only good things can happen if we extend our hands to our colleagues in LMICs and their patients in order to meet the unmet need and contribute to building a timely, safer, more equitable healthcare.

We are elated to comment positively on a successful twinning mission and also on the very first Global Neurosurgery themed article in Acta Neurochirurgica.

\section{References}

1. Barthélemy EJ, Park KB, Johnson W (2018) Neurosurgery and sustainable development goals. World Neurosurg 120:143-152

2. Dewan MC, Rattani A, Fieggen G et al (2019) Global neurosurgery: the current capacity and deficit in the provision of essential neurosurgical care. Executive summary of the Global Neurosurgery
Initiative at the Program in Global Surgery and Social Change. J Neurosurg 130:1055-1064

3. El-Husseiny H, Esene IN (2015) Training and research in neurosurgery: where are we? Neurosciences 20(3):300

4. Fuller A, Tran T, Muhumuza M et al (2016) Building neurosurgical capacity in low and middle income countries. eNeurologicalSci 3: $1-6$

5. Global neurosurgical workforce map https://www.wfns.org/menu/ 61/global-neurosurgical-workforce-map Accessed 14 Feb 2020

6. Intersurgeon www.intersurgeon.org Accessed 14 Feb 2020

7. Karekezi C, El Khamlichi A (2019) Takeoff of African neurosurgery and the World Federation of Neurosurgical Societies Rabat Training Center Alumni. World Neurosurgery 126:576-580

8. Karekezi C, El Khamlichi A et al (2020) The impact of Africantrained neurosurgeons on sub-Saharan Africa. Neurosurg Focus 48(3): $\mathrm{E} 4$

9. Kolias AG, Rubiano AM, Figaji A et al (2019) Traumatic brain injury: global collaboration for a global challenge. Lancet Neurol 18:136-137

10. Meara JG, Leather AJM, Hagander L et al (2015) Global surgery 2030: evidence and solutions for achieving health, welfare, and economic development. Lancet. 386:569-6241

11. Mukhopadhyay S, Punchak M, Rattani A et al (2019) The global neurosurgical workforce: a mixed-methods assessment of density and growth. J Neurosurg 130:1142-1148

12. Ng-Kamstra JS, Greenberg SLM, Abdullah F et al (2016) Global surgery 2030: a roadmap for high income country actors. BMJ Glob Health 1:e000011

13. Park KB, Johnson WD, Dempsey RJ (2016) Global neurosurgery: the unmet need. World Neurosurg 88:32-35

14. Robertson FC, Esene IN, Kolias AG et al (2019) Global perspectives on task shifting and task sharing in neurosurgery. World Neurosurg X:100060

15. Rosseau G, Johnson WD, Park KB et al (2020) Global neurosurgery: continued momentum at the 72nd World Health Assembly. J Neurosurg 1-5. https://doi.org/10.3171/2019.11.JNS191823

16. Servadei F, Rossini Z, Nicolosi F et al (2018) The role of neurosurgery in countries with limited facilities: facts and challenges. World Neurosurg 112:315-321

17. Uche EO, Wilfred MC, Ajuzieogu O (2020) Improving capacity and access to neurosurgery in sub-Saharan Africa using a twinning paradigm pioneered by the Swedish African neurosurgical collaboration. Acta Neurochir. https://doi.org/10.1007/s00701-019-04207-6

18. Venturini S, Park KB (2018) Evaluating the effectiveness and the impact of donated neurosurgical equipment on neurosurgical units in low- and middle-income countries: the world Federation of Neurosurgical Societies Experience. World Neurosurg 109:98-109

19. Vervoort D: The Visa conondrum in global health https://blogs.bmj. $\mathrm{com} / \mathrm{bmj} / 2019 / 06 / 21 /$ dominique-vervoort-the-visa-conundrum-inglobal-health/ Accessed 17 Jan 2020

20. WFNS Global Neurosurgery Committee http://globalneurosurgery. org Accessed 3 Feb 2020

21. Wiafe BA, Karekezi C, Jaklitsch MT et al (2020) Perspectives on surgical oncology in Africa. Eur J Surg Oncol 46:3-5

Publisher's note Springer Nature remains neutral with regard to jurisdictional claims in published maps and institutional affiliations. 